\title{
THE RESEARCH ON THE SPECTRAL CHARACTERISTICS OF SEA FOG BASED ON CALIOP AND MODIS DATA
}

\author{
Wan Jianhua ${ }^{1, *}$, Su Jing ${ }^{1}$,Liu Shanwei ${ }^{1}$,Sheng Hui ${ }^{1}$ \\ ${ }^{1}$ School of Geosciences, China University of Petroleum (East China),Qingdao,China,s16010067@ s.upc.edu.cn
}

Commission III, WG III / 8

KEY WORDS : Sea fog, Low Cloud, Caliop, Modis, Monitoring, Spectral Characteristics

\begin{abstract}
:
In view of that difficulty of distinguish between sea fog and low cloud by optical remote sensing mean, the research on spect ral characteristics of sea fog is focused and carried out . The satellite laser radar CALIOP data and the high spectral MODIS data were obtained from May to December 2017, and the scattering coefficient and the vertical height information were extracted from the atmospheric attenuation of the lower star to extract the sea fog sample points, and the spectral response curve based on MODIS was formed to analyse the spectral response characteristics of the sea fog, thus providing a theoretical basis for the monitoring of sea fog with optical remote sensing image.
\end{abstract}

\section{INTRODUCTION}

Sea fog is a common severe weather phenomenon in China's coastal areas, especially in the yellow sea. When there is a sea fog, the condensation of water vapor produces a large number of droplets suspended above the sea surface. The scattering and reflection of the fog droplets reduce the visibility of the sea surface and have a significant impact on human sea activities(Zhang et al,2009) Sea fog has brought great harm to sea navigation, fishery production, offshore oil development, Marine military activities and human life and health(Deng et al,2013)

In recent years, the research on the use of remote sensing to detect sea fog has been carried out. In 2005, Bendix et al. analyzed the optical characteristics of fog by using data from 7 channels of Terra MODIS, demonstrating the feasibility of using MODIS data for fog detection(Bendix $\mathbf{J}$ et al,2004,2005). In 2011, wu, et al., found that CALIOP was more likely to distinguish clouds than MODIS, which is also because CALIOP is more sensitive to thin clouds(Wu et al,2011). In 2015, lu bo et al. conducted a study on the detection method of sea fog using CALIOP data, and proposed the method of detecting the sea fog with the data of the CALIOP secondary VFM product and the first-order backscatter coefficient data( $\mathrm{Lu}$ et al,2015). The problem with the current study of the sea fog is that the spectrum of the sea fog and the low cloud is similar, and it is difficult to distinguish between the sea fog and the low cloud when detecting the range of sea fog.It is clear that the spectral response of sea fog is a prerequisite for the study of sea fog detection using remote sensing data. However, there are few studies on the spectral response characteristics of sea fog, so this paper intends to use CALIOP and MODIS data to study the spectral response characteristics of sea fog.

\section{THE S TUDY AREA AND THE DATA}

\subsection{The Study Area}

The yellow sea and bohai are adjacent to the coastal fog area of the northwest Pacific Ocean (Lewis J M,2004), which is the most frequent sea area in China. The sea fog in the yellow sea and the bohai has a wide range of influence (generally extended to inland area), and the concentration is large (The date on which the visibility is less than $200 \mathrm{~m}$ frequency up to 87\%) (Xi,1996), which has a serious impact on the traffic and production life of the sea and coastal areas. This article selects the study area for the yellow and bohai seas, latitude and longitude range of $30^{\circ} \mathrm{N}$ $-42^{\circ} \mathrm{N}, 118^{\circ} \mathrm{E}-128^{\circ} \mathrm{E}$. Figure 1 shows the scope of the study area. 


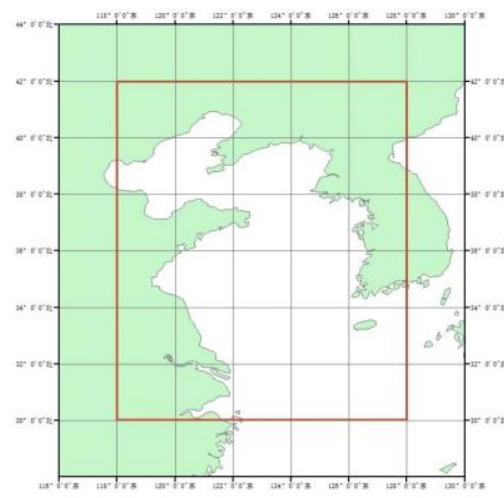

Figure 1. The study area

\subsection{The date}

The data used in this article is the calipso lidar CALIOP data and the hyperspectral remote sensing image MODIS data. The CALIOP data mainly contains the information of atmospheric profiles under the stars with high vertical resolution, which can effectively distinguish aerosol, sea fog, low cloud, medium and high layer cloud in atmospheric types, and obtain high information of all types.Moreover, the CALIOP data has a unique data structure. At different altitudes, it can obtain different resolution information, and the higher the altitude, the higher the resolution. When the altitude is below $8.2 \mathrm{~km}$, the radial resolution can reach $30 \mathrm{~m}$, and the horizontal resolution can reach $333 \mathrm{~m}$ (Sun,2014). MODIS data has 36 bands with high temporal resolution and high spatial resolution. The Aqua satellite carried by MODIS has the same orbit as CALIPSO, and the time difference between the two is only $73 \mathrm{~s}$ (Wei,2013). Therefore, it can be considered that they are synchronous observation, so MODIS can be combined with CALIOP data to detect the spectral response characteristics of sea fog.

According to the data of the atmospheric sounding station in the area around yellow sea and bohai, which is provided by the exploration data website of the university of Wyoming, the humidity data of Qingdao, dalian, white feathers, wushan, guangzhou, jeju, and suny ang are analyzed in combination with the satellite cloud map, so that there may be a sea fog when relative humidity is greater than $90 \%$, so as to screen the date of the possibility of sea fog in 2017.

\section{DATA PREPROCESS ING}

The CALIOP data is operated directly by MATLAB without preprocessing..The preprocessing of MODIS L1 data includes three parts: geometric correction, radiometric calibration and Solar zenith correction.

After MODIS L1 data has been radiometrically corrected, some data overlaps and shifts between two scan lines, commonly known as the "double eyelid" phenomenon, and the more it overlaps and shifts to the scanning edge, the more serious it is(Zhang,2009).therefore, the "double eyelid" phenomenon needs to be handled when MODIS data is applied. The correction method used in this paper is ground control point
(GCP) correction. Known pixel coordinates of the original space, triangular linear transformation relationship may be determined corrected spatial coordinates of the corresponding point. Using GCP data, the geometric distortion process of the original satellite image is simulated, and a corresponding relationship between the original distortion image space and the standard space (correction space) for geographic mapping is established. This correspondence is used to transform all the elements in the distorted space into the correction image space to achieve geometric precision correction.

MODIS L1 data to save storage space, the floating-point data through the offset (offset) and scale factor (scale) is converted to a 16-bit integer, so the need for radiation calibration data conversion to get a practical significance of the reflectivity value And radiance value.

Reflectance radiometric calibration equation (Zhou et al,2010)is:

$R_{B}=$ Reflectance_Scale $_{B} *\left(S I_{B}-{\left.\text { Reflectance_off } \text { set }_{B}\right)}_{(1)}\right.$

Among them, $S I_{B}$ is the count value of a channel point, Reflectance_Scale $e_{B}$ and Reflectance_offset ${ }_{B}$ are the proportional coefficient and offset of the reflectivity channel, stored in the attribute domain of the data file, and can be viewed.

During the satellite-to-ground scanning, the solar altitude angles at different detection points are different, which results in a certain deviation between the radiation and reflection detection values of the underlying surface and the true values. In order to eliminate this kind of deviation, the sun elevation angle correction must be performed on the MODIS data. Only the solar zenith angle product is used in the MODIS data, so the solar zenith angle is converted according to the following formula(Zhou et al,2010):

$$
\mathrm{z}_{\mathrm{S}}=D N * S_{-} \text {Scale } * \pi / 180
$$

Among them, $\mathrm{Z}_{\mathrm{S}}$ is the arc corresponding to the zenith angle, and DN is the solar zenith angle of a certain point. S_Scale is the scaling ratio between the probe value and the actual value. The default value is 0.01 .

Reflectance correction formula(Zhou et al,2010):

$$
\mathrm{SR}_{B}=\mathrm{R}_{\mathrm{B}} / \cos \left(\mathrm{Z}_{\mathrm{S}}\right)
$$

Among them, $\mathrm{SR}_{B}$ is the solar zenith angle corrected reflectivity, $R_{B}$ is the calibrated reflectivity, $Z_{S}$ is the solar zenith angle.

\section{SEA FOG S AMPLE POINT IDENTIFICATION}

In the identification of sea fog experiments in this paper, the hyperspectral MODIS data match the space-borne laser radar CALIOP data, combined with height information, the point under the stars, the sea fog, atmospheric profile of aerosol altostratus clouds,, to extract the sea fog sample points. Due to the particle characteristics of sea fog, the Mie scattering effect of 
sea fog is very obvious, and the backscattering coefficient of attenuation at CALIOP $532 \mathrm{~nm}$ channel is large, close to $0.1 . \mathrm{n}$ the vertical height information of CALIOP L1, the height of the cloud bottom is less than $50 \mathrm{~m}$, the height of the low cloud is greater than $50 \mathrm{~m}$, the height of the cloud top is less than $1000 \mathrm{~m}$, and the height of the middle cloud is greater than $1000 \mathrm{~m}$.

Remove aerosols based on the atmospheric classification of CALIOP VFM data. According to a $532 \mathrm{~nm}$ channel after the atmospheric attenuation to the scattering coefficient and vertical height information, in this paper, the scattering coefficient is greater than $0.03 \mathrm{~km}^{-1} \mathrm{sr}^{-1}$ and the cloud base height below $50 \mathrm{~m}$ is extracted as sea fog. The CALIOP star point attenuation backscatter coefficient is greater than $0.03 \mathrm{~km}^{-1} \mathrm{sr}^{-1}$ and the cloud base height is greater than $50 \mathrm{~m}$ The cloud top height is less than $1000 \mathrm{~m}$ is extracted as a low cloud.The mid-high cloud was extracted by subtracting the CALIOP sub-satellite point with a backscatter coefficient greater than $0.03 \mathrm{~km}^{-1} \mathrm{sr}^{-1}$ and a cloud base height greater than $1000 \mathrm{~m}$. A sample database based on MODIS data is established to match the identified sea fog, low cloud and medium high cloud sample points with MODIS data.

Take the case of a sea fog in the yellow bohai sea on May 13, 2017. FIG. 2 and 3 show the scattering coefficient profile of the atmospheric attenuation of CALIOP 532nm band. Figure 4 is an RGB image synthesized from MODIS data. As shown in figure 2, as shown in the red box within $31^{\circ} \mathrm{N}-34.7^{\circ} \mathrm{N}$, star point near the sea level under $532 \mathrm{~nm}$ attenuation after to scattering coefficient greater than $0.03 \mathrm{~km}^{-1} \mathrm{sr}^{-1}$, the discriminant for the low clouds and the sea fog. Green and yellow box frame, the cloud base height is greater than $2 \mathrm{~km}$, high cloud is determined. In the blue frame, the cloud base height is greater than $50 \mathrm{~m}$, and the height of the cloud is less than $1000 \mathrm{~m}$, which is considered as low cloud. In Figure 3, the red line is the height of the cloud top, the blue line is the height of the cloud base, $30^{\circ} 50^{\prime} \mathrm{N}-31^{\circ}$ $54^{\prime} \mathrm{N}$, and the cloud base height is greater than $50 \mathrm{~m}$, and it is judged as a low cloud. The sea fog was judged at $32^{\circ} \mathrm{N}-32^{\circ} 18^{\prime}$ $\mathrm{N}$ and $33^{\circ} \mathrm{N}-34^{\circ} 36^{\prime} \mathrm{N}$ with a cloud base height of less than $50 \mathrm{~m}$. In Figure 4, the yellow line is the CALIOP running trajectory. Matching the discriminant results to the MODIS image results in the sub-satellite point data type:The range of the purple box is low clouds, the range of the powder box is medium and high clouds, the range of the blue box is sea fog, the range of the green box is sea fog and high-neutral clouds, and the range of the red box is low clouds and high clouds.

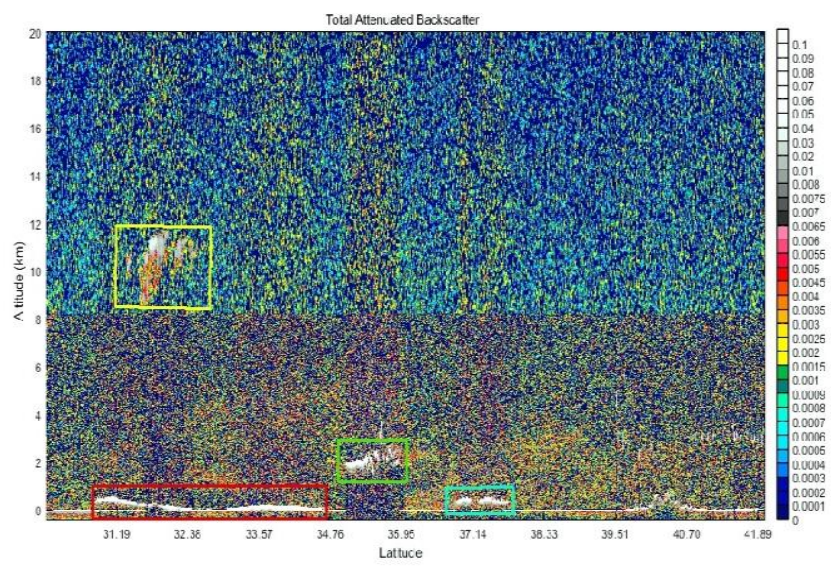

Figure 2. May 13th, 2017 CALIOP 532nm Atmospheric Attenuation Backscattering Coefficient Profile

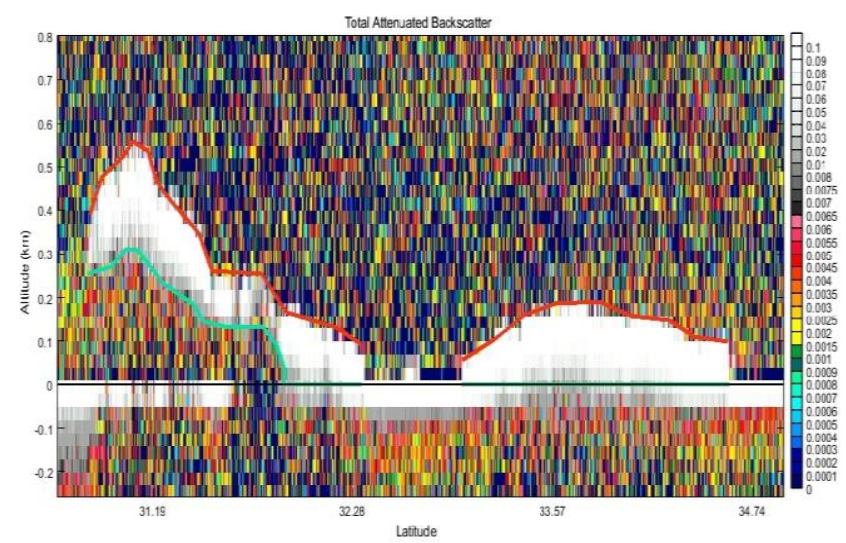

Figure 3. May 13th, 2017 CALIOP 532nm Atmospheric Attenuation Backscattering Coefficient Profile 


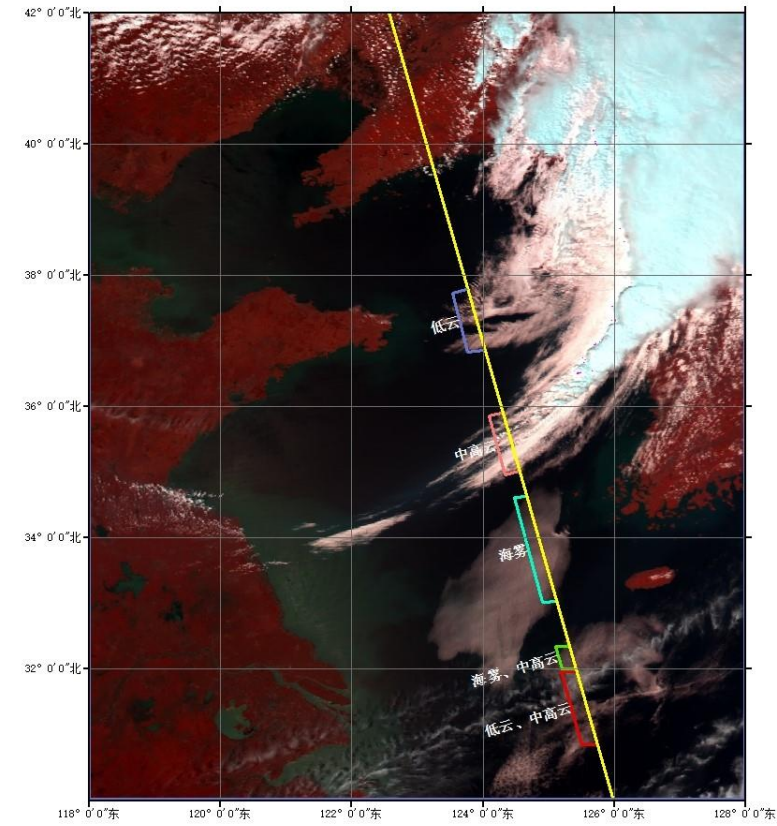

Figure 4. May 13, 2017 MODIS RGB Images

\section{SEA FOG S PECTRAL CHARACTERIS TICS ANALYS IS}

The sea mist identification is carried out by means of the CALIOP based sea fog recognition algorithm, and the sea fog low cloud, medium height cloud, clear air sea surface identified by the CALIOP data is matched with the reflectance band and the radiance brightness band data of the aqua-modis, and the sample database is set up. Statistical information of MODIS bands in the sample area is generated, and spectral response curves such as sea fog and low clouds are generated. Spectral response characteristics of visible fog, near, mid, and far infrared wave fog are analyzed. Fig. 5 shows the spectral response curves of sea fog, low cloud and clear sea in the reflectivity band.

The reflectance band spectral response curve following conclusions:

Firest, there is a large number of invalid values in the band 6, 10, 11, 12, 13lo, 13hi, 14lo, 14hi, 15 and 16, which cannot be applied to the study of sea fog identification. In the 8th and 9th bands, there are some invalid values. In this band, the reflectivity of sea fog is greater than that of low clouds, and it is still necessary to further prove the study of sea fog recognition in the 8th and 9th bands.

Secend, in the visible and near infrared bands, the reflectivity of the low clouds is the highest, and the reflectivity of the chart is the lowest. Although the reflectivity spectrum curve of sea fog is similar to the spectral curve of low clouds, the reflectivity value of each band is less than the reflectivity value of the low cloud.
Thirdly, in the range of band 1 to band 5 , the reflectivity of sea fog is less than 0.3 , and the reflectivity of low clouds is greater than 0.3 . The reflectivity of the chart is less than 0.1 in addition to the third and 8th bands, namely the blue band and the violet light.

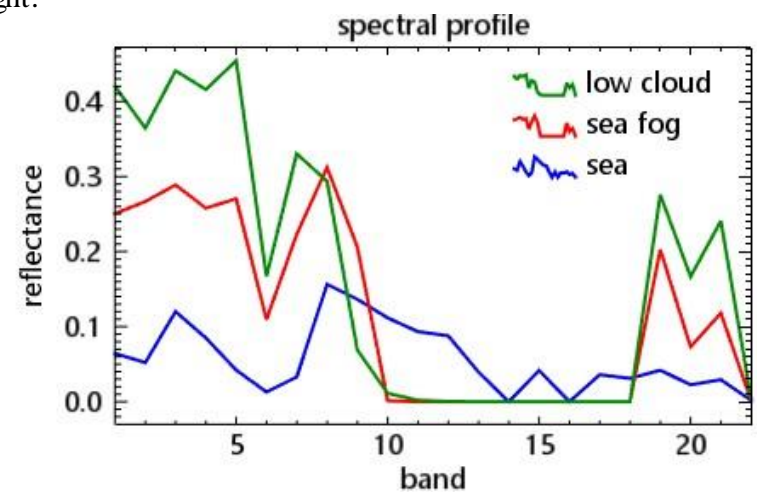

Figure 5. spectra characterization of MODIS reflectance bands

\section{CONCLUSION}

This paper, by using spaceborne lidar CALIOP data in combination with high spectral MODIS data using under the star point of backscatter coefficient and vertical height information to distinguish the sea fog and clouds, sea fog, clouds, sea surface samples, formed the sea fog, clouds, sea surface in the spectral response of the MODIS reflectance spectrum characteristic curve. According to the sea fog, low cloud on the MODIS reflectance of each band numerical transformation, with the size of the filter can effectively distinguish between the sea of mist and cloud band, for the following based on the study of optical remote sensing image of sea fog.

\section{ACKNOWLEDGEMENTS}

This research has been supported by National Key R\&D Program of China (2017YFC1405600), National Natural Science Foundation of China (41776182) and Shandong Provincial Natural Science Foundation of China (ZR2016DM16).

\section{REFERENCES}

Bendix J, Thies B and Cermak J,2004. Fog Detection With Terra-Modis And Msg-Seviri[C]// Proceedings of the 2003 Meteorological Satellite Conference.PP:427—435

Bendix J, Thies B and Cermak J,2005. Ground Fog Detection from Space Based on MODIS Daytime Data-A Feasibility Study [J]. Weather \& Forecasting.20(6):989-1005.

Deng Y J,Wang J C and Cao J,2013. Detection of daytime fog in south china sea using modis data. Journal of tropical meteorology. 29(6):1046-1050.

Huang B, Chen T, Chen $\mathrm{Y}$ and Deng X T. Study on the numerical forecasting system and test method of Huang Haihai's sea fog $[\mathrm{J}]$. Meteorological Science and Technology, 2009, 37(3):271-275. 
$\mathrm{Lu} \mathrm{B,2015.} \mathrm{Caliop} \mathrm{sea} \mathrm{fog} \mathrm{detection} \mathrm{and} \mathrm{its} \mathrm{application} \mathrm{to} \mathrm{the}$ daytime modis remote sensing of sea fog. Ocean University of China.

Lewis J M. Sea Fog Research in the United Kingdom and United States: A Historical Essay Including Outlook[J]. Bulletin of the American Meteorological Society, 2004, 85(3):395-408.

Xi X X.The main features of Qingdao and its offshore sea fog [J]. Ocean bulletin, 1996(3):87-91.

Wu D, Hu Y and Mccormick M P,2011. Global cloud-layer distribution statistics from 1 year CALIPSO lidar observations[J]. International Journal of Remote Sensing. 32(5):1269-1288

Zhou J f, Zhou Y L, Li S H and Ding K. Radiation calibration of MODIS L1B data and correction of solar zenith angle[J]. Journal of Southwest Forestry University, 2010, 30(3):77-81.

Zhang J W, Zhang S P, Wu X J, Liu Y C and Liu J W,2009. The research on yellow sea sea fog based on modis data: sea fog properties retrieval and spatial- temporal distribution. Journal of Ocean University of China (Natural Science Edition) Natural Science Edition, 2009 (s1): 311-318.

Zhang $\mathrm{J}$ w. Research on sea fog in the Yellow Sea based on MODIS [D]. Ocean University of China, 2009. 